\title{
Monitoring of the Human Body Signal through the Internet of Things (IoT) Based LoRa Wireless Network System
}

\author{
Mohammad Shahidul Islam ${ }^{1, *}$, Mohammad Tariqul Islam ${ }^{1, * \mathbb{D}}$, Ali F. Almutairi ${ }^{2, *(D)}$, \\ Gan Kok Beng ${ }^{1}$, Norbahiah Misran ${ }^{1}$ (D) and Nowshad Amin ${ }^{3}$ \\ 1 Centre of Advanced Electronic and Communication Engineering, Universiti Kebangsaan Malaysia, \\ Bangi 43600, Malaysia; gankokbeng@ukm.edu.my (G.K.B.); bahiah@ukm.edu.my (N.M.) \\ 2 Electrical Engineering Department, Kuwait University, Kuwait City 13060, Kuwait \\ 3 Institute of Sustainable Energy, Universiti Tenaga Nasional (@The National Energy University), Jalan \\ IKRAM-UNITEN, Kajang 43000, Selangor, Malaysia; nowshad@uniten.edu.my \\ * Correspondence: p97645@siswa.ukm.edu.my (M.S.I.); tariqul@ukm.edu.my (M.T.I.); \\ ali.almut@ku.edu.kw (A.F.A.); Tel.: +60-19-366-6192 (M.T.I.)
}

Received: 4 March 2019; Accepted: 30 April 2019; Published: 8 May 2019

Featured Application: The development process of an IoT based human health monitoring system has been described in this paper. This paper also analyzed the collected biomedical sensors data with normal human body data to prove the systems' performance, effectiveness, and efficiency.

\begin{abstract}
Internet of Things (IoT) based healthcare system is now at the top peak because of its potentialities among all other IoT applications. Supporting sensors integrated with IoT healthcare can effectively analyze and gather the patients' physical health data that has made the IoT based healthcare ubiquitously acceptable. A set of challenges including the continuous presence of the healthcare professionals and staff as well as the proper amenities in remote areas during emergency situations need to be addressed for developing a flexible IoT based healthcare system. Besides that, the human entered data are not as reliable as automated generated data. The development of the IoT based health monitoring system allows a personalized treatment in certain circumstances that helps to reduce the healthcare cost and wastage with a continuous improving outcome. We present an IoT based health monitoring system using the MySignals development shield with (Low power long range) LoRa wireless network system. Electrocardiogram (ECG) sensor, body temperature sensor, pulse rate, and oxygen saturation sensor have been used with MySignals and LoRa. Evaluating the performances and effectiveness of the sensors and wireless platform devices are also analyzed in this paper by applying physiological data analysis methodology and statistical analysis. MySignals enables the stated sensors to gather physical data. The aim is to transmit the gathered data from MySignals to a personal computer by implementing a wireless system with LoRa. The results show that MySignals is successfully interfaced with the ECG, temperature, oxygen saturation, and pulse rate sensors. The communication with the hyper-terminal program using LoRa has been implemented and an IoT based healthcare system is being developed in MySignals platform with the expected results getting from the sensors.
\end{abstract}

Keywords: IoT healthcare; biomedical sensors; MySignals; LoRa

\section{Introduction}

A continuous and long-term healthcare system is essential in the healthcare environment nowadays to make the involved process efficient and reliable; to provide peace of mind to the patients by ensuring 
complete medical care. Internet of Things (IoT) is a fast-growing worldwide network of interconnected variety of objects that supports many input-output devices, sensors, and actuators based on standard communication protocol [1,2]. Many applications are in the development process because of the IoT potentialities, of which quite a few numbers of IoT applications are being deployed or developed for our society. These autonomous quality IoT applications are helping to improve our daily lives [3,4]. In the near future, IoT for healthcare will become a stable solution for the healthcare system. The IoT has a diversity of application domains in which IoT for healthcare is still at the top of the research because of its potentiality [5]. Besides, developing technologies of IoT such as (Radio frequency identification) RFID, wireless network technologies (Bluetooth Low Energy, Wi-Fi, ZigBee), and (Low power wireless area network) LPWAN technologies like LoRa and SigFox are promising in terms of the IoT application development in a large scale and they are contributing to improving the device connection to the internet as well as the efficiency of the IoT application operation.

Nowadays, one of the top challenges that every country is facing is healthcare. In developing countries, the feasible solution for reducing the expenditures of chronic diseases and disabilities is the healthcare system because health organizations are transforming themselves into more efficient, coordinated, and user-centered systems [6,7]. To address the health issues, the Internet of Things (IoT) offers a world of networked devices, cloud-based applications, and services, with diverse cooperation mechanisms based on the confluence of a right standardization, efficient wireless protocols, improved sensors, cheaper and low-power microprocessors, and wireless technologies [8-10]. Besides, LPWAN technologies are taking place to enable new human-centric health and wireless monitoring applications [11]. LoRa is one of such LPWAN protocols, which targets deployments where end-devices have limited energy (for example, battery-powered) with not transmitting more than a few bytes at a time and where data traffic can be initiated either by the end-device or by an external entity wishing to communicate with the end-device $[12,13]$.

Table 1 represents the characteristics of different network technologies for IoT. It is noticeable that LoRa has a high communication range with lower data rate, which is the best choice for IoT based healthcare system because of its specification that ensures the perfect interoperability between the IoT objects. In this paper, we present an IoT based healthcare system with MySignals platform, which is an e-health monitoring development platform working in conjunction with the Arduino Uno and it can connect and measure up to fifteen sensors. MySignals with LoRa wireless network system is applied to different biomedical sensors. The performances and effectiveness of the gathered data have also been analyzed that can be used for future purposes. The LoRa communication with the multiprotocol radio shield has been applied for the gathered sensor data.

Table 1. Topology and frequency of the communication technologies [14-17].

\begin{tabular}{ccc}
\hline Network Technologies & Topology & Radio Frequency (MHz) \\
\hline BLE & Adhoc & $2.4 \mathrm{GHz}$ \\
ZigBee & Mesh & $868.3 \mathrm{MHz}, 902-928 \mathrm{MHz}, 2.4 \mathrm{GHz}$ \\
Wi-Fi & Star & $2.4 \mathrm{GHz}$ \\
SigFox & Star & $862-928 \mathrm{MHz}$ \\
LoRa & Star/Mesh & $433,860-1020 \mathrm{MHz}$ \\
\hline
\end{tabular}

\section{Related Work}

IoT based healthcare system performs all the healthcare activities including diagnosing, monitoring, and remote surgeries by connecting to all available resources over the internet [18]. IoT based healthcare system can be separated into three areas of intervention such as clinical care, remote monitoring, and context-awareness, where clinical care is for the hospitalized patient who needs continuous and close attention, remote monitoring is for the elderly or child patient who needs to come to the hospital or clinic on a regular basis for a health checkup, and context-awareness is for identifying the patients' overall condition from anywhere in terms of clinical assistance, respectively [19]. In recent years, 
many researchers have proposed and developed IoT based healthcare systems in various manners, where all the development architectures follow the typical format directly or indirectly. A two-stage fundamental approach has been proposed for a remote healthcare monitoring system, where the first stage is to gather the sensors data and the second stage is to send the data using a Femto-LTE network [20]. Body Sensor Network (BSN) technology is also one of the core technologies in an IoT based health monitoring system. To support rapid and flexible prototyping and management of a body sensor network, an open source programming framework named SPINE (signal processing in node environment) has been proposed that efficiently addresses the identified requirements of the hardware or software sensor platforms [21]. Later, a novel framework for collaborative body sensor network (CBSN) named C-Spine has been proposed, where it supports multi-sensor data fusion among CBSNs to enable time-dependent data integration, filtering, and classification [22]. To prove the effectiveness of the C-Spine framework, a system named e-Shake has been introduced that can detect the emotions of two meeting people by allowing a ECG-based heart rate monitoring component to analyze the heart rate variability. The e-Shake detects three kinds of different emotional meetings like no emotion, emotion from one individual, and emotion from two individuals. Figure 1 represents the CBSN system e-Shake graphical user interface (GUI) during the handshake detection.

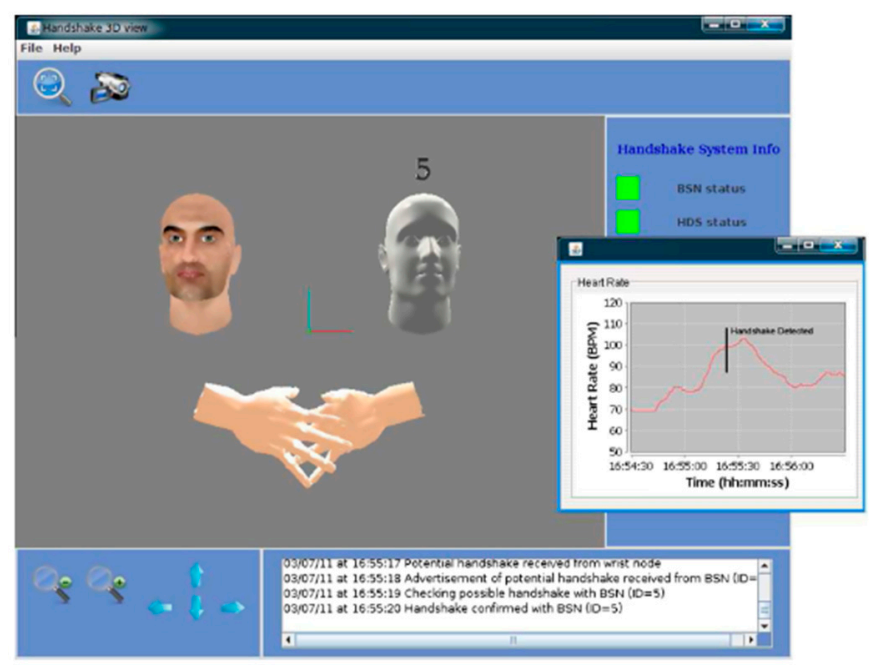

Figure 1. e-Shake graphical user interface (GUI) during handshake detection event [22].

An IoT based fog computing approach with a prototype of smart e-health gateway shown in Figure 2 has been implemented by Rahmani et al. [23], which investigated the range of high-level services offered by a smart gateway to sensors and end users [24]. A fog-based system for IoT has been presented in reference [25], where human body signals such as ECG, body temperature, and contextual data such as room temperature, humidity, and air quality can be monitored in real time. A Wi-Fi based ECG monitoring system has been presented by Shebi et al. [26], where the ECG sensor data are displayed to the smart-phone after performing the wireless transmission. Wearable ECG monitoring system has also been proposed that uses a ZigBee wireless network [27] that is shown in Figure 3. A wireless blood pressure monitoring system using Bluetooth technology is also developed to display the systolic and diastolic blood pressure value. A pilot study to validate the performance of the developed prototype has also been performed [28] that is shown in Figure 4. 


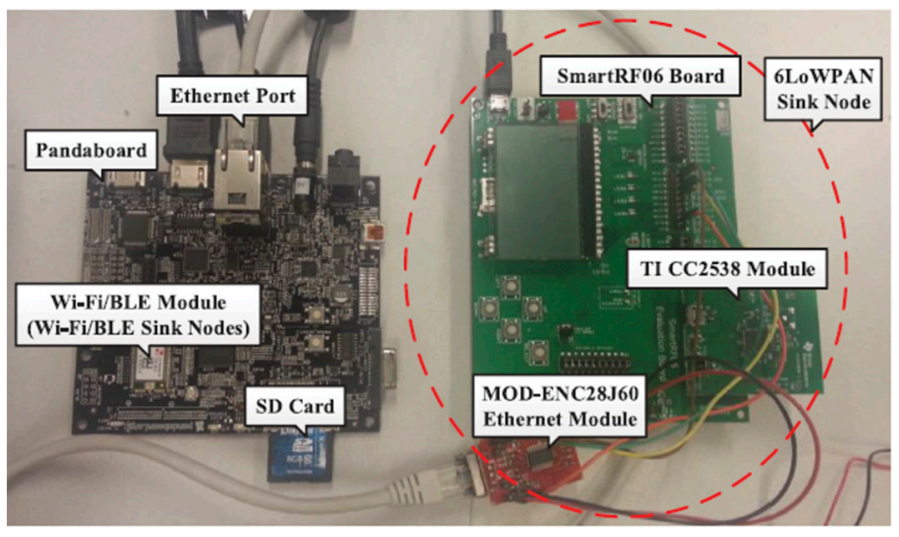

Figure 2. e-health gateway [23].
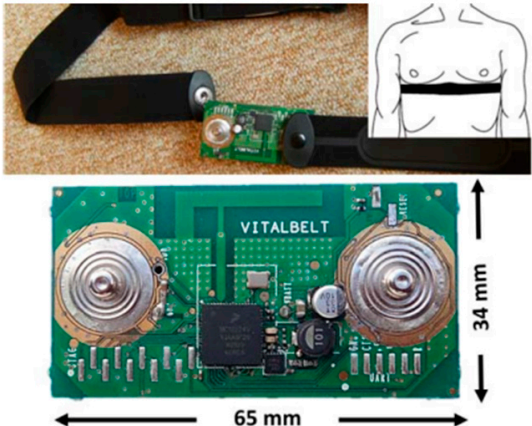

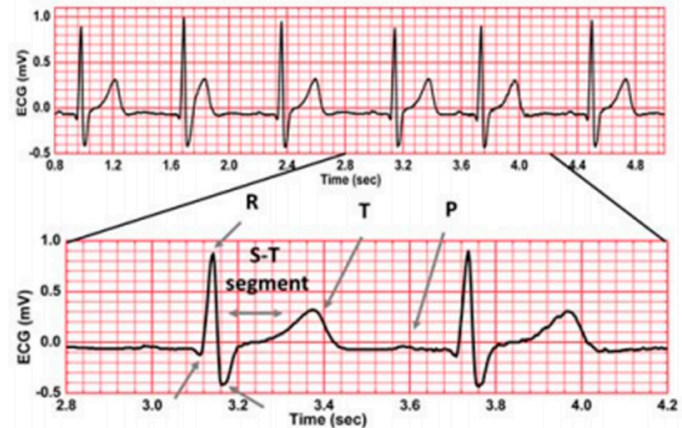

Figure 3. Signal waveform by ZigBee [27].
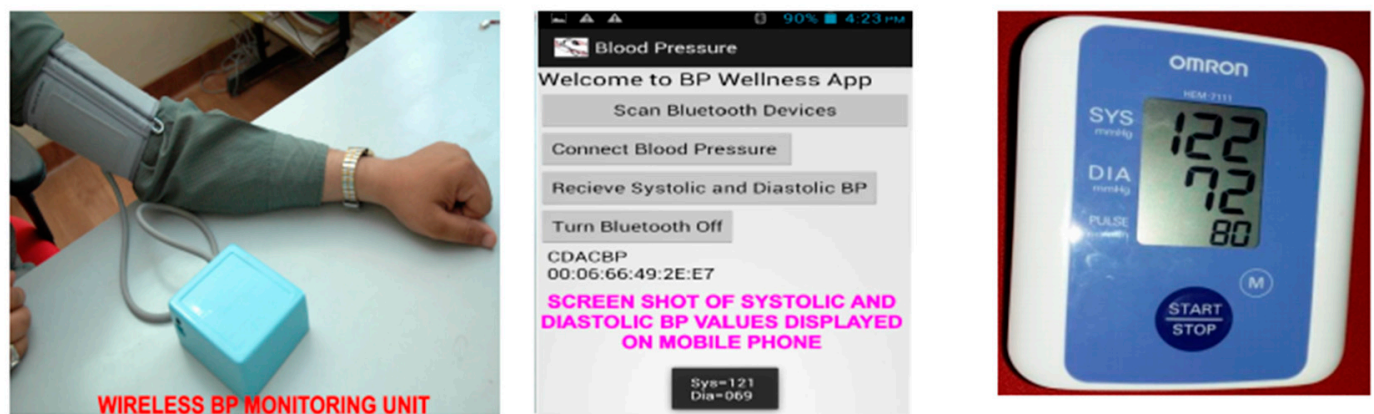

Figure 4. Systolic Blood Pressure (SBP) and Diastolic Blood Pressure (DBP) using prototype by Bluetooth [28].

Besides, LoRa is always different compared to other short-range sensor network technologies such as Bluetooth, ZigBee, Wi-Fi, etc., and it provides a unique set of features including a wide area connectivity for low power and low data rate devices. The existing IoT based healthcare issues including high-cost communication links with $3 \mathrm{G} / 4 \mathrm{G}$, data privacy, and ignorance of monitored health parameters is now a matter of concern. Many deployments and proposals about the solution of these issues have been made where it is shown that LoRa has the ability to solve all of these issues by integrating medical sensors, cloud, and gateways. LoRa in mesh networking for a large area monitoring application has been developed by Lee et.al [29]. They deployed 19 LoRa sensors in mesh networking devices to identify the Packet Delivery Ratio (PDR) by comparing it with a star-topology networking device. The result shows that mesh networking devices achieved $88.49 \%$, where it is $58.7 \%$ for star-topology networking devices. A proposal has been made by Mdhaffar et al. [30], indicating that the usage of LoRa in the medical sector to monitoring a patient can solve the aforementioned issues. Three steps are proposed including getting the patient physical metrics from medical sensors, transmit the data through LoRa sensors and gateway, and send the data to the cloud for further proceedings of 
medical records. Diabetes and arterial hypertension have been measured through this system but it is not capable of getting continuous medical data that hinders the evaluation of ECG data. The proposed architecture that shows the LoRa sensors integrated IoT based healthcare shown in Figure 5.

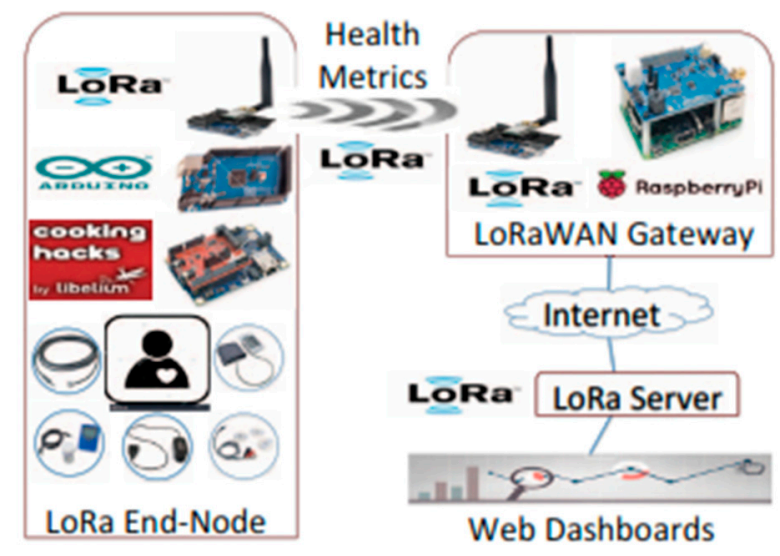

Figure 5. Based healthcare with low power long range (LoRa) [30].

Long coverage area is one of the main characteristics of LoRa. Many applications have been tested within large geographical and indoor areas. The performance of LoRa sensors in the indoor areas is measured by Petjjrvi et al. [31], where the target was to check the hypothesis of health monitoring applications. The sensor nodes are operated with various physical layer settings of LoRa sensors. The results of the experiment show that the sending data amount may differ up to 200-fold, which indicates the efficiency of selecting LoRa in terms of indoor health monitoring. For the large area coverage area, an aforementioned developed framework indicates that the data transmission works perfectly using the LoRa wireless network system with a low power consumption, although the security issues are not mentioned [29].

\section{Development Methodology}

Figure 6 represents the overall block diagram, where the development process of the health monitoring system for IoT is given. Biomedical sensors are connected to the MySignals and Arduino Uno to collect the health data with a wired and Bluetooth form. Arduino and MySignals are connected with LoRa by the multiprotocol radio shield. The specifications of MySignals are given below in Table 2 . It also represents the controlling and monitoring characteristics with different sensors and the data transmission line like wire/Bluetooth/WiFi/LoRa.

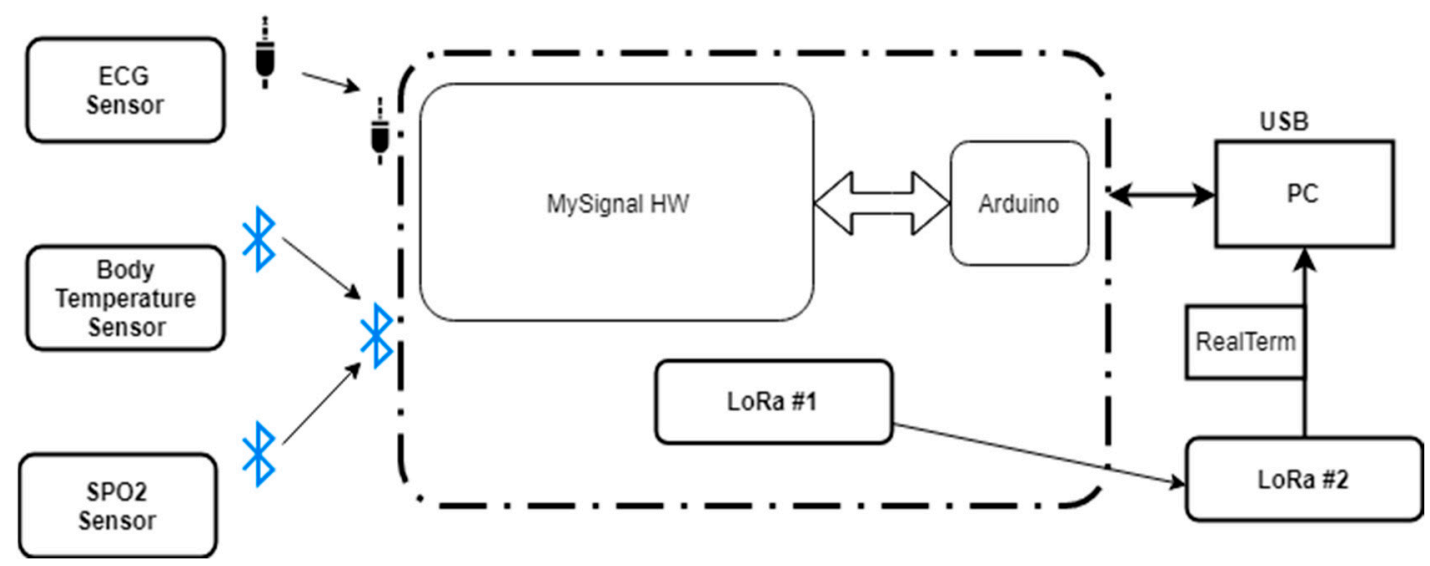

Figure 6. Block Diagram Representing the Overall System. 
Table 2. Specifications of MySignals. EMG: Electromyography; ECG: Electrocardiography.

\begin{tabular}{ccc}
\hline Control and Monitoring & Data Transmission & Applied with LoRa \\
\hline EMG signals & Wired + Bluetooth/WiFi/LoRa & $X$ \\
ECG signals & Wired + Bluetooth/WiFi/LoRa & $\sqrt{ }$ \\
Snore signals & Wired/Bluetooth/WiFi/LoRa & $X$ \\
Airflow control of user & Wired/Bluetooth/WiFi/LoRa & $X$ \\
Body temperature data & Wired/Bluetooth/WiFi/LoRa & $\sqrt{ }$ \\
Galvanic skin response & Wired/Bluetooth/WiFi/LoRa & $X$ \\
measurements & Wired/Bluetooth/WiFi/LoRa & \multicolumn{2}{|}{} \\
Body position detection & Wired/Bluetooth/WiFi/LoRa & $\sqrt{ }$ \\
Pulse and oxygen functions & Wired/Bluetooth & $X$ \\
Blood pressure control device & Wired/Bluetooth & $X$ \\
Glucometer monitor & Wired/Bluetooth/WiFi/LoRa & X \\
Spirometer monitor & Bluetooth & $X$ \\
Body Scale & &
\end{tabular}

Figure 7 is the diagram of MySignals platform with 12 sensor ports. This platform is also integrated with a WiFi serial transceiver module ESP8266. The collected data then transferred via the LoRa module and the Waspmote gateway to the personal computer. Table 3 represents the LoRa specifications that have been used in the proposed IoT healthcare project.

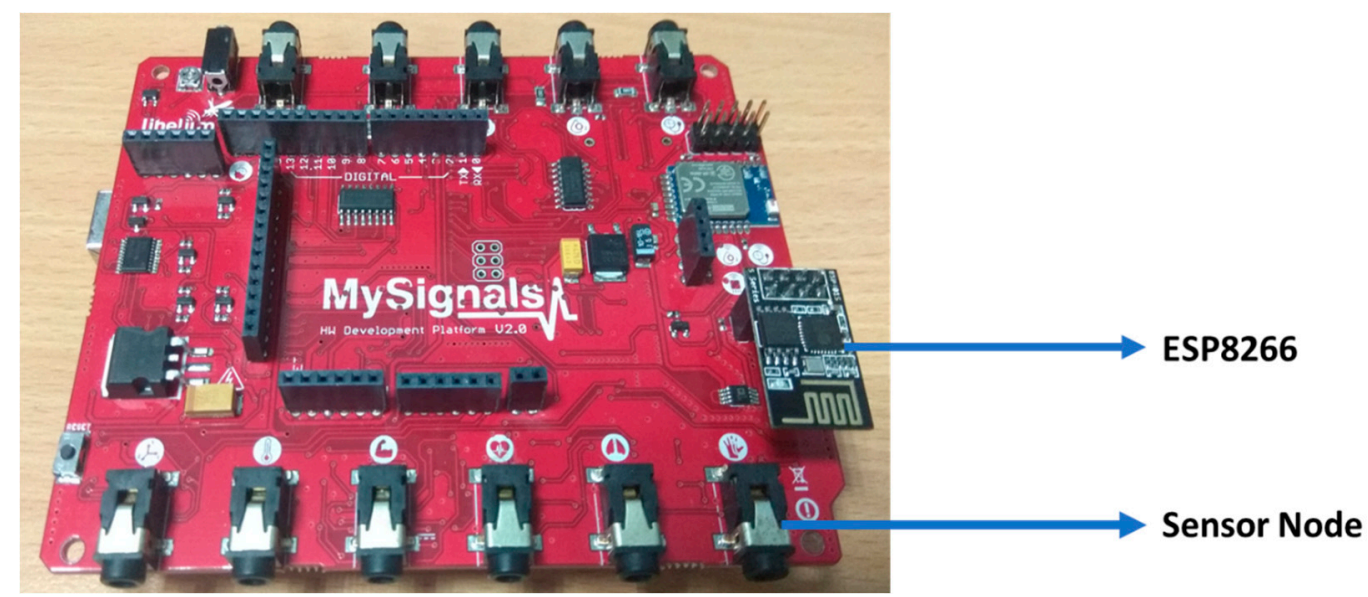

Figure 7. MySignals with Sensor Nodes and WiFi Module.

Table 3. Specifications of LoRa.

\begin{tabular}{cc}
\hline Characteristics & Specification \\
\hline Module & SX1272 \\
Dual Frequency Band & $902-928 \mathrm{MHz}(\mathrm{US})$ \\
Transmission Power & $25 \mathrm{~mW}$ \\
Sensitivity & $-134 \mathrm{dBm}$ \\
Channels & $13(900 \mathrm{MHz})$ \\
Range & LOS $=21 \mathrm{~km}(13.4 \mathrm{~m})$ \\
\hline
\end{tabular}

LoRa is connected with the multiprotocol radio shield, which is an interconnection shield for Arduino and designed to connect two communication modules at the same time. This multiprotocol radio shield can connect any Arduino wireless module like Bluetooth, WiFi, ZigBee, RFID, LoRa, etc. Figure 8 represents the diagram of LoRa with the multiprotocol radio shield with Waspmote. Waspmote is an ultra-low power $(7 \mu \mathrm{A})$ open source wireless sensor platform that is specially focused on the implementation of low consumption modes, which allows the sensor nodes ("motes") to be completely autonomous with its battery power. This Waspmote supports LPWAN technologies for 
$868 \mathrm{MHz}$ or $900 \mathrm{MHz}$ frequencies. One hyper-terminal named RealTerm, for capturing, controlling, and debugging binary and other difficult data streams, is used to connect with the Lora modules to send and receive packet data with a specific baud rate for confirmation that the sensor data are transferring successfully or not. Figure 9 shows the biomedical sensors that have been used in this proposed IoT healthcare system.

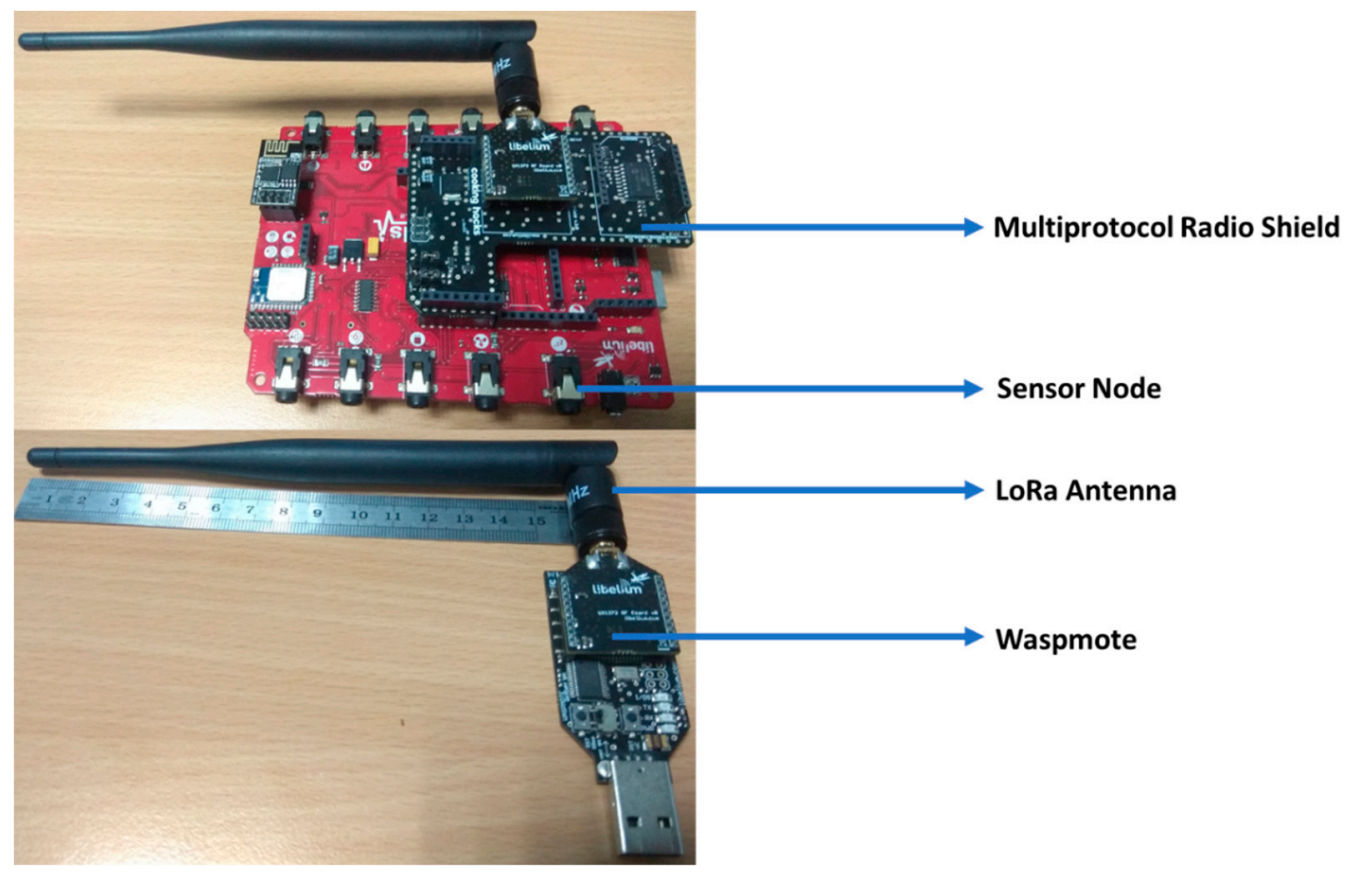

Figure 8. LoRa with Waspmote and a Multiprotocol Radio Shield.
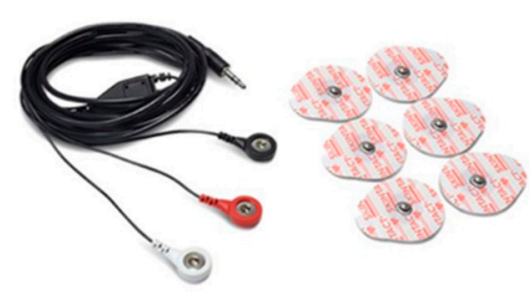

(a)

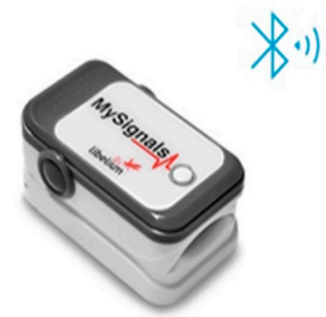

(b)

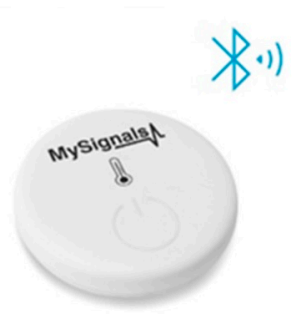

(c)

Figure 9. (a) ECG Sensor (b) pulse rate and oxygen saturation sensor $\left(\mathrm{SPO}_{2}\right)(\mathbf{c})$ body temperature sensor.

The physiological properties of the sensor equipment selected to be measured during the health monitoring experimental trails with the ECG sensor for heart rate, pulse rate, and oxygen saturation sensor and body temperature sensor. These metrics were selected based on the ability to predict a patient's health status and reliability of the automated data. Each physiological metric measurement with the definitions and units are given in Table 4 . The sensor data are evaluated by the following two methods. One is from the serial monitor of Arduino Uno and the other is from a hyper-terminal named RealTerm connecting with LoRa. The serial monitor gathers the sensor data via Bluetooth or wire (ECG) and the hyper-terminal gets the data from Arduino Uno connecting with LoRa modules, which are also shown in Figure 10. 
Table 4. Physiological metrics and information.

\begin{tabular}{ccc}
\hline Metric & Definition & Unit \\
\hline Heart Rate & Number of heart pulses a patient experiences per minute & Heart beats per minute (BPM) \\
Pulse rate & Number of heart beats of a patient experiences per minute & Heart beats per minute (BPM) \\
Oxygen saturation & Percentage of oxygen level in the patient's blood & Percentage $(\%)$ \\
Body Temperature & Temperature range in patient's body & Degree centigrade $\left({ }^{\circ} \mathrm{C}\right)$ \\
\hline
\end{tabular}

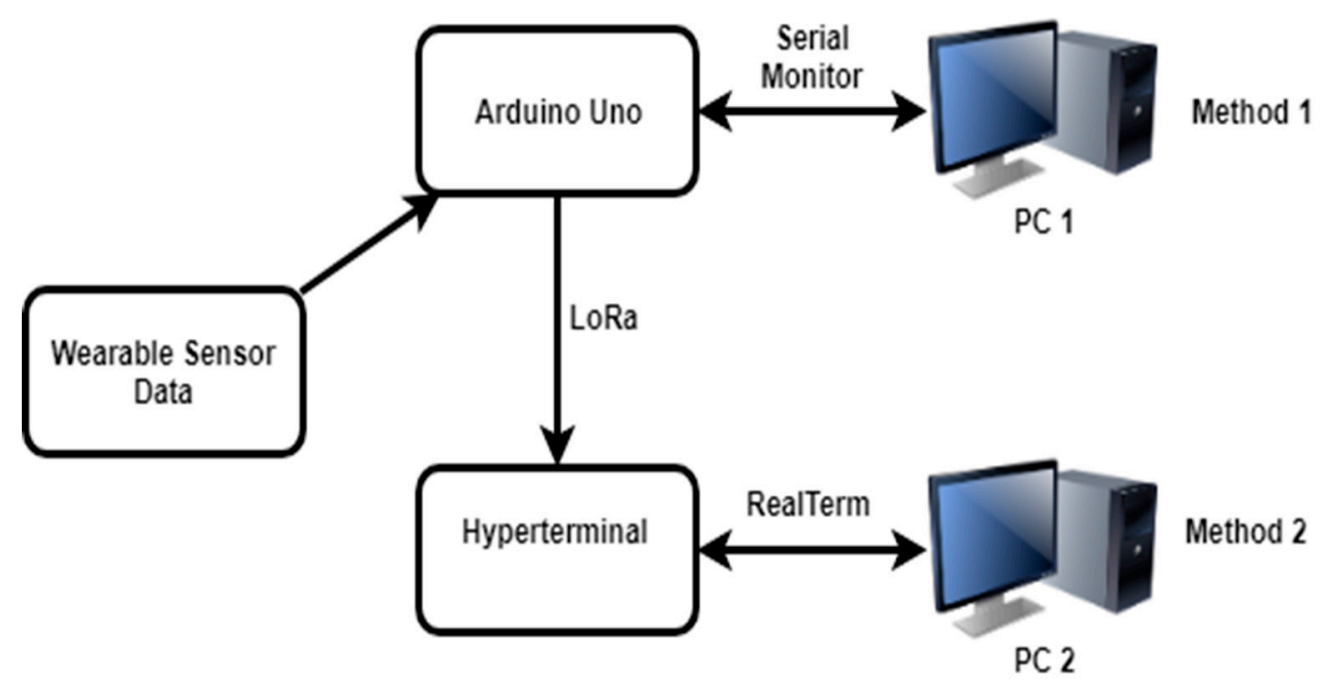

Figure 10. Physiological data analysis methodology.

\section{Results and Discussion}

Figure 11 presents the overall system with all the elements. The analysis is aimed at collecting human body-centric data and evaluating the performance and effectiveness of the sensors and the wireless platform to ensure the suitability and sustainability of the devices in an IoT based health monitoring system. Figure 12 presents the ECG sensor data, which is collected from the real human body. Figure 13 presents the body temperature, pulse rate, and oxygen saturation rate collected from the serial monitor of Arduino. The average body temperature gathered from the sensor was $35.40^{\circ} \mathrm{C}$. The pulse rate was 55 to $62 \mathrm{bpm}$ and the oxygen saturation rate was 96 to $100 \%$. Figure 14 represents the results displayed on RealTerm to read the serial data coming from the COM port that shows two different messages repeating, which are the Arduino interfaced with the multiprotocol shield and the LoRa module.

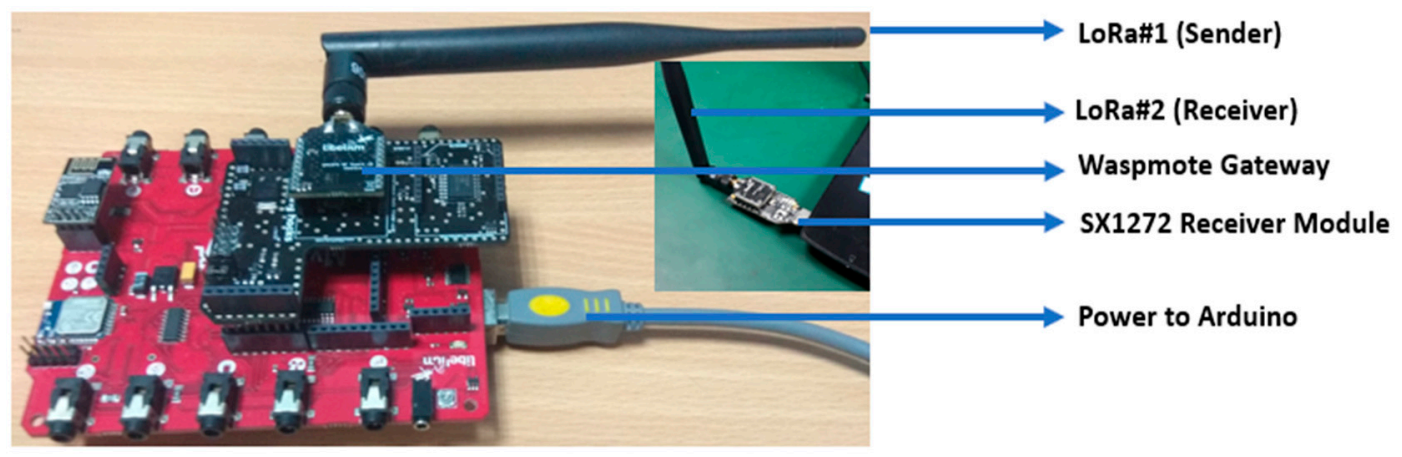

Figure 11. Overall connection of the system. 

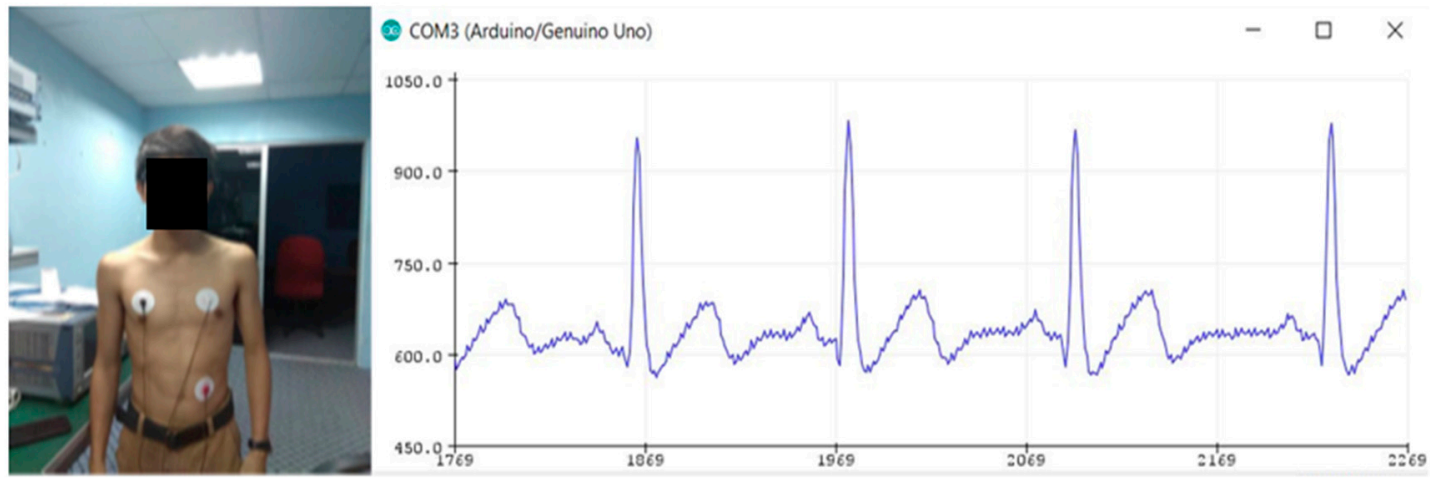

Figure 12. ECG sensor data.

сOM3 (Arduino/Genuino Uno)

।

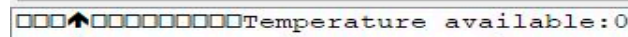

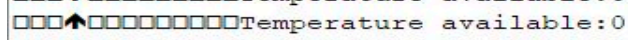

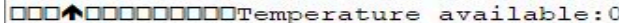

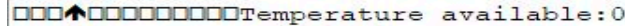

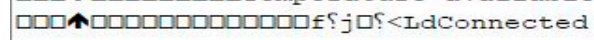

$\square \square+\square \square$ Temp : 35.10

Temp $: 35.10$

Temp: 35.20

Temp : 35.30

Temp: 35.30

Temp: 35.32

Temp: 35.34

Temp: 35.35

Temp: 35.38

Temp: 35.45

Temp: 35.45

(a)

(2) $\operatorname{COM} 3$ (Arduino/Genuino Uno)

|

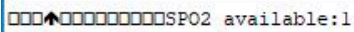

SPO2 found. Connecting

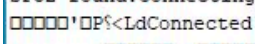

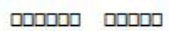

Sp02: 96\% Pulse: 62ppm

andisconnecting error code: 0

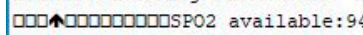

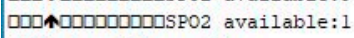

SPO2 found. Connecting

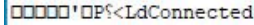

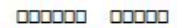

Sp02: $100 \%$ Pulse: $55 \mathrm{ppm}$

audisconnecting error code:

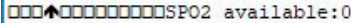

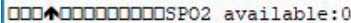

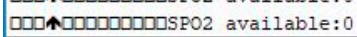

(b)

Figure 13. (a) Temperature and (b) $\mathrm{SpO}_{2}$ readings from serial monitor. 


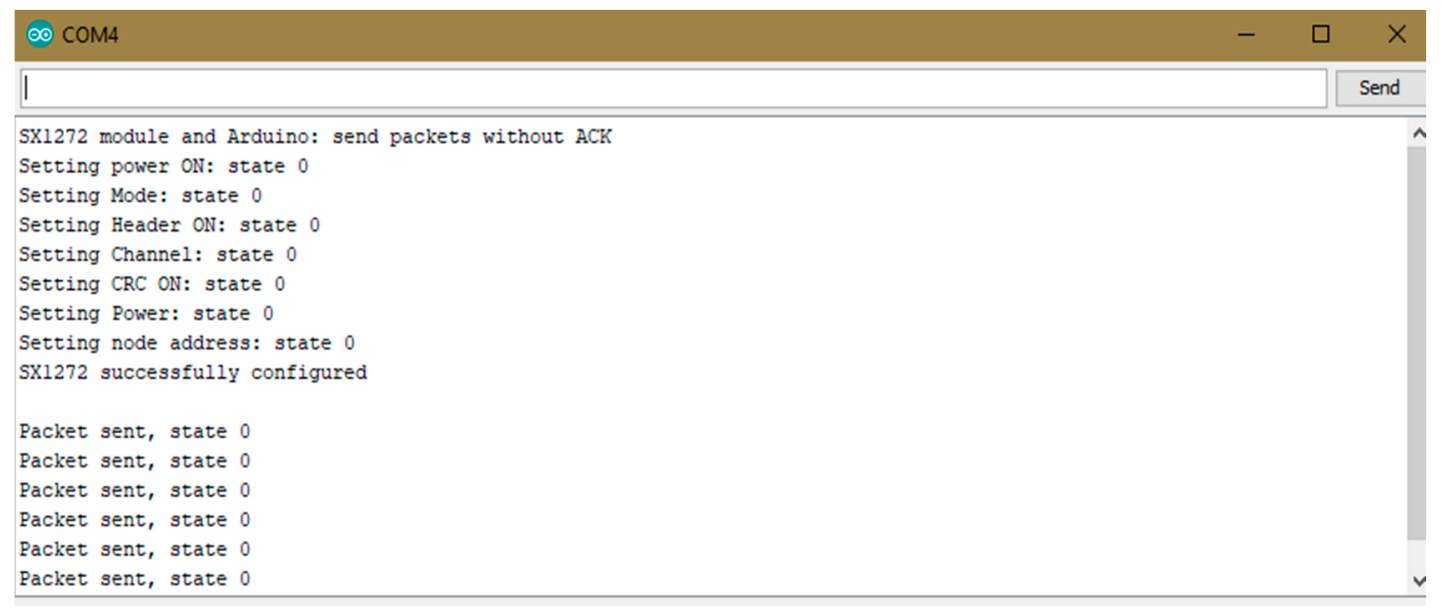

(a)

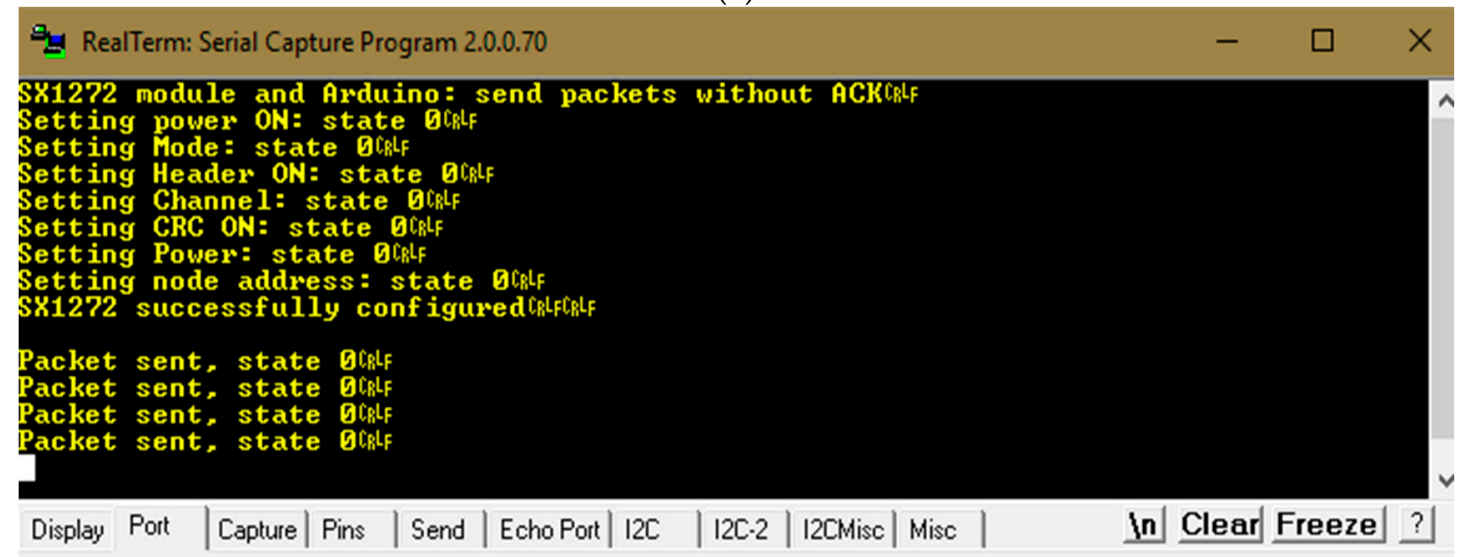

(b)

RealTerm: Serial Capture Program 2.0.0.70

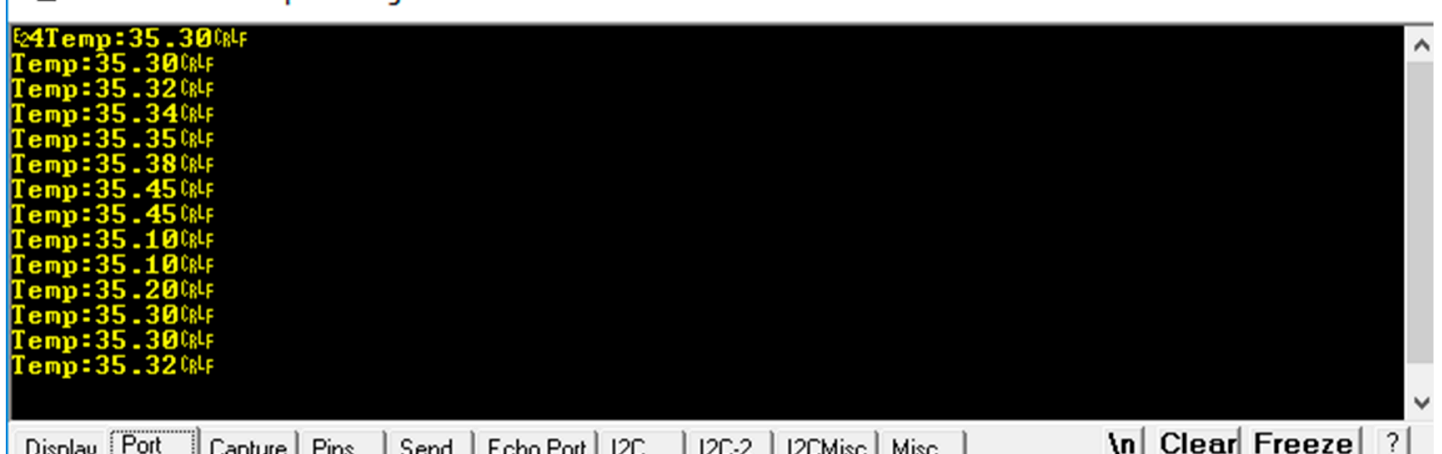

(c)

Figure 14. (a) LoRa module being set up before transmitting packets (b) test packets sending using RealTerm (c) receiving sensor data using RealTerm.

The overall analysis between the normal data of the human body and sensor data are presented in Table 5. It is noticeable that the pulse rate, oxygen saturation, and ECG values have no issue with the human body normal data except body temperature data. This has happened due to the experimental environment. The experiment was performed in the air-conditioned laboratory and that is why the temperature fell down a bit, although the sensor data were acceptable. 
Table 5. Analysis of the human body-centric data and gathered sensor data.

\begin{tabular}{cccc}
\hline $\begin{array}{c}\text { Human Body } \\
\text { Characteristics }\end{array}$ & Normal Data & Sensor Data (Proposed System) & Issues \\
\hline Body Temperature & $36.5-37.5^{\circ} \mathrm{C}$ & $35.10-35.40^{\circ} \mathrm{C}$ & $\begin{array}{c}\text { Air condition } \\
\text { environment } \\
\text { Pulse Rate }\end{array}$ \\
$60-100 \mathrm{bpm}$ & $96-99$ & $55-62 \mathrm{bpm}$ & No \\
Oxygen Saturation (\%) & $60-100 \mathrm{bpm}$ & $96-100$ & No \\
ECG Values (Heart Rate) & $0.1-0.2$ & $60-100 \mathrm{bpm}$ & No \\
ECG Values (Time & 0.1 & No \\
Interval (s)) & & & \\
\hline
\end{tabular}

Figures 15-17 represent the graph of gathered data from the body temperature sensor, pulse rate sensor, and oxygen saturation sensor, which continues with Table 6 that represents the sample data of wearable sensors with calculating results of the sample mean, standard deviation, and confidence interval approximations range. Confidence interval approximations range shows the data accuracy in a mathematical form where it is shown that pulse rate, and oxygen saturation sensor data have a $99 \%$ confidence interval, where it is $95 \%$ for body temperature sensor data. For the hypothesis testing shown in Table 7, we used the Left Tail method where the $\mathrm{z}$ score was above the significance level critical value. Body temperature sensor data and Pulse rate sensor data were within the non-rejection area, which means the hypothesis testing is accepted in terms of the gathered value. On the contrary, it was noticeable that the hypothesis testing was rejected in terms of the left tail method, which indicates that the pulse rate data are unable to maximize the accuracy.

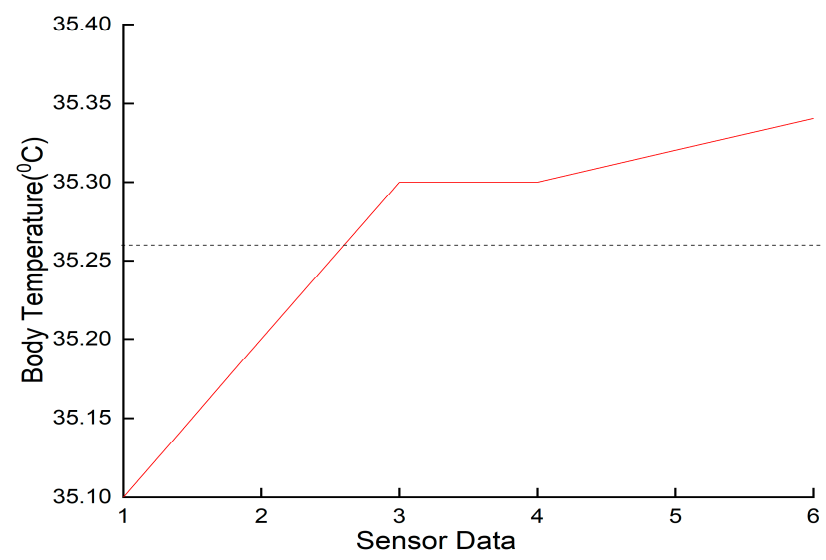

Figure 15. Body temperature data collected from the sensor.

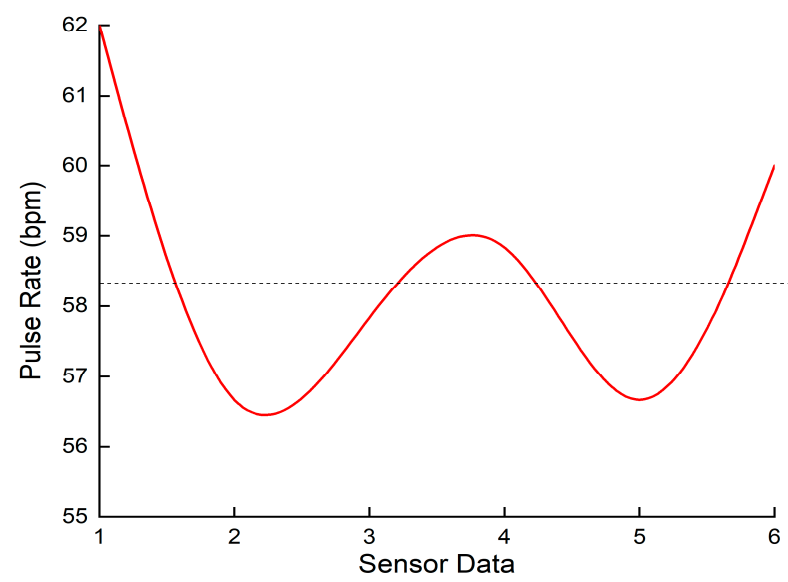

Figure 16. Pulse rate data collected from the sensor. 


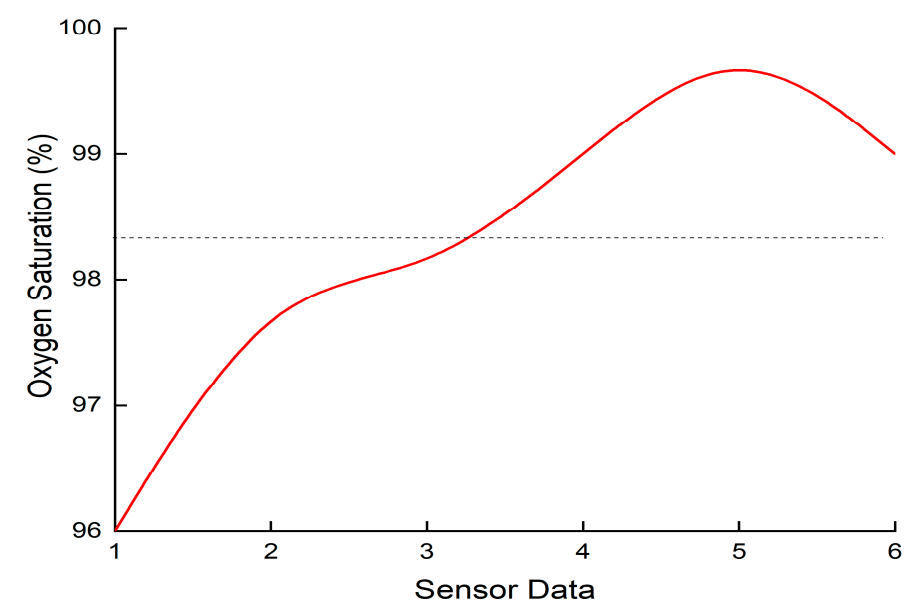

Figure 17. Oxygen saturation data collected from the sensor.

Table 6. Statistical analysis of sample sensor data.

\begin{tabular}{ccccc}
\hline Wearable Sensors & $\begin{array}{c}\text { Sample Mean } \\
\text { (Sensor Data) }\end{array}$ & $\begin{array}{c}\text { Population Standard } \\
\text { Deviation }\end{array}$ & $\begin{array}{c}\text { Sample Standard } \\
\text { Deviation }\end{array}$ & $\begin{array}{c}\text { Confidence Interval } \\
\text { Approximations Range }\end{array}$ \\
\hline Body Temperature & 35.26 & 0.084 & 0.092 & $35.18-35.33(95 \%)$ \\
Pulse rate & 58.33 & 2.624 & 2.875 & $53.30-61.35(99 \%)$ \\
Oxygen saturation & 98.33 & 1.247 & 1.366 & $96.89-99.77(99 \%)$ \\
\hline
\end{tabular}

Table 7. Hypothesis testing of sample sensor data.

\begin{tabular}{|c|c|c|c|c|c|}
\hline $\begin{array}{l}\text { Hypothesis Testing } \\
\text { (Left Tail) }\end{array}$ & $\begin{array}{l}\text { Null Hypothesis } \\
\text { Mean }\left(\mathrm{H}_{0}\right)\end{array}$ & $\begin{array}{l}\text { Sample Mean (Normal } \\
\text { Human Body) }\end{array}$ & Sample Size & $\begin{array}{l}\text { Significance } \\
\text { Value }\end{array}$ & Result \\
\hline $\begin{array}{l}\text { Body Temperature } \\
\text { Sensor }\end{array}$ & 35.26 & 37 & 6 & $5 \%$ & $\begin{array}{l}\text { The null hypothesis } \\
\text { cannot be rejected. The } \mathrm{z} \\
\text { score of } 46.33 \text { is within } \\
\text { the nonrejection area }\end{array}$ \\
\hline Pulse Rate Sensor & 58.33 & 80 & 6 & $5 \%$ & $\begin{array}{l}\text { The null hypothesis } \\
\text { cannot be rejected. The } \mathrm{z} \\
\text { score of } 18.46 \text { is within } \\
\text { the nonrejection area }\end{array}$ \\
\hline $\begin{array}{l}\text { Oxygen Saturation } \\
\text { Sensor }\end{array}$ & 98.33 & 97.5 & 6 & $5 \%$ & $\begin{array}{l}\text { The null hypothesis is } \\
\text { rejected and accepts the } \\
\text { alternative hypothesis. } \\
\text { The } z \text { score of }-1.49 \text { is in } \\
\text { the rejection area }\end{array}$ \\
\hline
\end{tabular}

LoRa module node class sends the message to the gateway for a certain period and the gateway receives it for further processing. LoRa $900 \mathrm{MHz}$ ISM (Industrial, scientific and medical) band has been used and the data rate is $12.5 \mathrm{kbps}$ with spreading factor 8 based on the specifications. Sensors related to measuring the vital body signal data such as the ECG sensor, body temperature sensor, oxygen saturation, and pulse rate sensor have the data rate of $8-10 \mathrm{kbps}$, which is an acceptable data transmission rate for LoRa [32]. In addition, the acceptable baud rate of LoRa is up to 115,200 bps. The transmitting and receiving baud rate must be similar for proper transmission, which has also been used in the project for sensor data transfer. For ECG data, the continuous data flow has been shown in the serial plotter of Arduino and it is not possible to send the data in plotter form. However, it is possible to send in a form of listing like the serial monitor of Arduino Uno even though that data rate will be high for lesser samples.

The duty cycle of LoRa is often less than $1 \%$. If many devices send their packets at the same time, there might be a problem with packet loss or network overload. However, the data packets can be sent properly if every device is configured to send at different times, which is an undeniable flaw of the LoRa module. In this paper, the data flow shown by the sensor going through the LoRa and the Waspmote gateway has been taken at different times on a single channel. 
The main energy consumers are the microcontroller unit (Arduino Uno), sensor unit (MySignals), and RF unit (LoRa). Digital sensors e.g., MySignals are operated by the battery and have a low power consumption. The microcontroller Arduino Uno has the DC (Direct Current) per I/O pin is $40 \mathrm{~mA}$ which is taken as a reference to calculate the overall power consumption of data transmission of LoRa. The transmission power of the Waspmote SX1272 is $14 \mathrm{dBm}$. In this paper, the LoRa transmission and receiving range in terms of distance has been analyzed. The approximate data are shown in Table 8. It is noticeable that the transmission and receiving time is high due to the increased distance. It also increases the energy consumption rate due to the range and slower data rate. With a minimum range, the data rate is faster and shows lesser energy consumption. In general, more time transmitting means more energy consumption. The secure communication protocol is another important factor of every IoT system solutions, where the data transmission must be reliable, confidential, and available by preventing cyber-attacks. This IoT protocol is divided into three levels: Physical access, network and service and application levels. Every level consists of security issues and solutions. BLE (Bluetooth Low Energy) has the data transit attack in its physical layer and Black Network is the new solution for this issue. MQTT (Message Queuing Telemetry Transport) and IPv4 both have the same data transit attack in their application, and network levels, however, secure MQTT with ABE (Attribute Based Encryption) and IPsec protocol are the new and standard solutions of these issues [33,34]. In this paper, the focus is on the LoRa wireless network system and it also has similar protocol levels. Data transit attacks are the main security issue in physical, network, and application levels of the proposed LoRa based solution. To solve the issue, Semtech Corporation, the development company of LoRa, has come up with a solution of embedded end-to-end (Advanced Encryption Standard) AES-128 encryption. This AES-128 is also known as an Application Key (AppKey) that encrypts all the data by ensuring optimal privacy and protection. In addition, the AppKey generates two session keys named Network Session Key (NwkSKey), which ensures the message integrity by creating a specific signature for every device and Application Session Key (AppSKey), which is used for both the encryption and decryption of the payload of the application data.

Table 8. Range versus transmission time/consumption. ACK: Acknowledgement.

\begin{tabular}{|c|c|c|c|c|c|c|c|}
\hline $\begin{array}{l}\text { Channel } \\
\text { Number }\end{array}$ & $\begin{array}{l}\text { Central } \\
\text { Frequency }\end{array}$ & $\begin{array}{l}\text { Sensitivity } \\
\text { (dB) }\end{array}$ & Range (m) & $\begin{array}{c}\text { Transmission } \\
\text { Time (ms) for a } \\
\text { 100-Byte Packet } \\
\text { Sent } \\
\text { (Approximate) }\end{array}$ & $\begin{array}{c}\text { Power } \\
\text { Consumption } \\
\text { (mA-ms/1000) } \\
\text { (Reference = } \\
40 \mathrm{~mA} \text { in TX } \\
\text { Mode) }\end{array}$ & $\begin{array}{c}\text { Transmission } \\
\text { Time (ms) for a } \\
\text { 100-Byte Packet } \\
\text { Sent and ACK } \\
\text { Received } \\
\text { (Approximate) }\end{array}$ & Comments \\
\hline CH_01_900 & $\begin{array}{l}905.24 \\
\mathrm{MHz}\end{array}$ & -134 & 1 & 700 & 28 & 1500 & $\begin{array}{c}\text { Minimum range, } \\
\text { Fast data rate, } \\
\text { Minimum battery } \\
\text { impact }\end{array}$ \\
\hline CH_01_900 & $\begin{array}{l}905.24 \\
\mathrm{MHz}\end{array}$ & 58.33 & 3 & 1000 & 40 & 1800 & $\begin{array}{c}\text { Maximum range, } \\
\text { Slow data rate, } \\
\text { Maximum battery } \\
\text { impact }\end{array}$ \\
\hline
\end{tabular}

Table 9 represents the comparison among the network technologies in terms of data rate, transmission range, power consumption, and battery life. It is noticeable that LoRa has the low power consumption and highest battery life compared to others. LoRa also has the highest transmission range even though it has a lower data rate compared to Wi-Fi or Bluetooth. Table 10 is presenting the comparison of different IoT based health monitoring systems in terms of applied sensor nodes, microcontroller, and power consumption. It is observed that the proposed system has applied the highest number of sensor nodes and it has a low power consumption compared to the systems who have used Wi-Fi. By analyzing the comparison tables, it can be said the proposed system has the highest compatibility compared to other network technology-based health monitoring system. 
Table 9. Comparison among network technologies. BLE: Bluetooth Low Energy; TX: Transmitter.

\begin{tabular}{ccccc}
\hline $\begin{array}{c}\text { Network } \\
\text { Technology }\end{array}$ & Data Rate & $\begin{array}{c}\text { Transmission } \\
\text { Range }\end{array}$ & $\begin{array}{c}\text { Power } \\
\text { Consumption }\end{array}$ & Battery Life \\
\hline BLE & $1-2 \mathrm{Mb} / \mathrm{s}$ & $10 \mathrm{~m}$ & $\begin{array}{c}40 \mathrm{~mA} \mathrm{TX} \text {, Standby } \\
0.2 \mathrm{~mA}\end{array}$ & 1 Year \\
ZigBee & $0.02-0.25 \mathrm{Mb} / \mathrm{s}$ & $100 \mathrm{~m}$ & $\begin{array}{c}30 \mathrm{~mA} \mathrm{TX}, \text { Standby } \\
1 \mu \mathrm{A}\end{array}$ & Week \\
Wi-Fi & $11 \mathrm{Mb} / \mathrm{s}-10$ & $<1 \mathrm{~km}$ & $\begin{array}{c}400 \mathrm{~mA} \mathrm{TX}, \\
\text { Standby } 20 \mathrm{~mA}\end{array}$ & Hours \\
& $\mathrm{Gb} / \mathrm{s}$ & & $28-44 \mathrm{~mA} \mathrm{TX}$, & 10 Years \\
LoRa & $290 \mathrm{bps}-50$ & $15 \mathrm{~km}$ & Standby $1.4 \mathrm{~mA}$ & \\
\hline
\end{tabular}

Table 10. Comparison among different health monitoring systems with applied sensor nodes. BLE: Bluetooth Low Energy; ECG: Electrocardiogram.

\begin{tabular}{|c|c|c|c|c|c|}
\hline Reference & System & Sensor Node & $\begin{array}{l}\text { Applied Network } \\
\text { Technology }\end{array}$ & Micro-Controller & $\begin{array}{c}\text { Power } \\
\text { Consumption }\end{array}$ \\
\hline [28] & $\begin{array}{l}\text { Blood Pressure } \\
\text { Monitoring } \\
\text { System }\end{array}$ & Blood Pressure & BLE & AT89c2051 & Low Power \\
\hline [35] & $\begin{array}{l}\text { ECG Telemetry } \\
\text { System }\end{array}$ & ECG & BLE & Arduino & Low Power \\
\hline [36] & $\begin{array}{l}\text { Remotely } \\
\text { Patient } \\
\text { Monitoring } \\
\text { System }\end{array}$ & Motion, ECG & BLE & MSP430 & Low Power \\
\hline [37] & $\begin{array}{c}\text { Health } \\
\text { Telemonitoring } \\
\text { System }\end{array}$ & $\begin{array}{l}\text { ECG, Blood } \\
\text { Pressure }\end{array}$ & BLE, ZigBee & N/A & $\begin{array}{l}\text { Low Power, } \\
\text { Potential } \\
\text { Battery-less }\end{array}$ \\
\hline [27] & $\begin{array}{c}\text { ECG } \\
\text { Monitoring } \\
\text { System }\end{array}$ & ECG & ZigBee & ARM7 & $\begin{array}{l}\text { Potential } \\
\text { Battery-less }\end{array}$ \\
\hline [38] & $\begin{array}{c}\text { Healthcare } \\
\text { System of } \\
\text { Doppler Shifts }\end{array}$ & $\begin{array}{c}\text { ECG, } \\
\text { Photo-plethysmogram } \\
\text { (PPG) }\end{array}$ & $\mathrm{Wi}-\mathrm{Fi}$ & N/A & High \\
\hline [39] & $\begin{array}{l}\text { Patients' Daily } \\
\text { Activity } \\
\text { Monitoring } \\
\text { System }\end{array}$ & Inertial & Wi-Fi & N/A & High \\
\hline [40] & $\begin{array}{l}\text { Safety and } \\
\text { Health } \\
\text { Monitoring } \\
\text { Applications }\end{array}$ & $\begin{array}{l}\text { PPG, Body } \\
\text { Temperature }\end{array}$ & LoRa & $\begin{array}{c}\text { ARM Cortex } \\
\text { M0 }\end{array}$ & Low Power \\
\hline [41] & $\begin{array}{c}\text { Sustainable } \\
\text { Health } \\
\text { Monitoring } \\
\text { System with } \\
\text { Smart Clothing }\end{array}$ & $\begin{array}{l}\text { ECG, Oxygen } \\
\text { Saturation, } \\
\text { Pulse Rate }\end{array}$ & Bluetooth & Arduino & Low Power \\
\hline Proposed & $\begin{array}{l}\text { Human Body } \\
\text { Signal } \\
\text { Monitoring } \\
\text { System }\end{array}$ & $\begin{array}{l}\text { ECG, Body } \\
\text { Temperature, } \\
\text { Pulse Rate, } \\
\text { Oxygen } \\
\text { Saturation }\end{array}$ & LoRa & Arduino Uno & Low Power \\
\hline
\end{tabular}

\section{Conclusions}

This paper presented an overall monitoring system of human body signal that is performed by biomedical sensors, MySignals, and the LoRa wireless network system. The MySignals platform is successfully interfaced with ECG, temperature, pulse rate, and oxygen saturation sensors to produce 
vital signs and data for medical patients or applications. The LoRa module has been installed and configured successfully to enable communications with a terminal program. To prove the effectiveness and efficiency of the sensor data, statistical analysis with hypothesis testing was performed. Overall, the performance of the system was effective in terms of collecting human body data, which is a suitable solution for IoT based healthcare.

Author Contributions: M.S.I. made significant contributions to this study regarding conception, design, analysis, and writing the draft manuscript. M.T.I. revised the article critically for important intellectual contents and supervised the whole study. A.F.A. participated in the development idea with a critical analysis and contributed to the write-up and revision of the manuscript. G.K.B., N.M., and N.A. revised the article.

Funding: This research was funded by Universiti Kebangsaan Malaysia, grant number DIP-2018-018.

Conflicts of Interest: The authors have declared that no competing interests exist.

\section{References}

1. Elkhodr, M.; Shahrestani, S.; Cheung, H. Emerging Wireless Technologies in the Internet of Things: A Comparative Study. Int. J. Wirel. Mob. Netw. 2016, 8, 67-82. [CrossRef]

2. Rohokale, V.M.; Prasad, N.R.; Prasad, R. A cooperative Internet of Things (IoT) for rural healthcare monitoring and control. In Proceedings of the 2011 2nd International Conference on Wireless Communication, Vehicular Technology, Information Theory and Aerospace \& Electronic Systems Technology (Wireless VITAE), Chennai, India, 28 February-3 March 2011.

3. Atzori, L.; Iera, A.; Morabito, G. The Internet of Things: A survey. Comput. Netw. 2010, 54, 2787-2805. [CrossRef]

4. Nordin, R.; Mohamad, H.; Behjati, M.; Kelechi, A.H.; Ramli, N.; Ishizu, K.; Kojima, F.; Ismail, M.; Idris, M. The world-first deployment of narrowband IoT for rural hydrological monitoring in UNESCO biosphere environment. In Proceedings of the 2017 IEEE 4th International Conference on Smart Instrumentation, Measurement and Application (ICSIMA), Putrajaya, Malaysia, 28-30 November 2017; pp. 1-4.

5. Darshan, K.R.; Anandakumar, K.R. A comprehensive review on usage of Internet of things (IoT) in healthcare system. In Proceedings of the 2015 International Conference on Emerging Research in Electronics, Computer Science and Technology (ICERECT), Mandya, India, 17-19 December 2015.

6. Jimenez, F.; Torres, R. Building an IoT-aware healthcare monitoring system. In Proceedings of the 201534 th International Conference of the Chilean Computer Science Society (SCCC), Santiago, Chile, 9-13 November 2015; pp. 5-8.

7. Yuen, M.-C.; Chu, S.Y.; Hong Chu, W.; Shuen Cheng, H.; Lam Ng, H.; Pang Yuen, S. A low-cost IoT smart home system. Int. J. Eng. Technol. 2018, 7, 3143-3147.

8. Fernandez, F.; Pallis, G. Opportunities and challenges of the Internet of Things for healthcare. In Proceedings of the 2014 4th International Conference on Wireless Mobile Communication and Healthcare-Transforming Healthcare Through Innovations in Mobile and Wireless Technologies (MOBIHEALTH), Athens, Greece, 3-5 November 2014; pp. 263-266.

9. Samy, G.N.; Ahmad, N.A.; Salwana, E.; Hassan, N.H.; Maarop, N.; Drus, S.M. Proposed Conceptual Iot-Based Patient Monitoring Sensor for Predicting and Controlling Dengue. Int. J. Grid Distrib. Comput. 2018, 11, 127-134.

10. Dauwed, M.A.; Yahaya, J.; Mansor, Z.; Hamdan, A.R. Human factors for IoT services utilization for health information exchange. J. Theor. Appl. Inf. Technol. 2018, 96, 2095-2105.

11. Petäjäjärvi, J.; Mikhaylov, K.; Hämäläinen, M.; Iinatti, J. Evaluation of LoRa LPWAN technology for remote health and wellbeing monitoring Evaluation of LoRa LPWAN Technology for Remote Health and Wellbeing Monitoring. In Proceedings of the 2016 10th International Symposium on Medical Information and Communication Technology (ISMICT), Worcester, MA, USA, 20-23 March 2016; pp. 1-5.

12. Augustin, A.; Yi, J.; Clausen, T.; Townsley, W.M. A study of Lora: Long range \& low power networks for the internet of things. Sensors (Switzerland) 2016, 16, 1466.

13. Ismail, N.L.; Kassim, M.; Ismail, M.; Mohamad, R. A review of low power wide area technology in licensed and unlicensed spectrum for IoT use cases. Bull. Electr. Eng. Inform. 2018, 7, 183-190. 
14. Ismail, D.; Rahman, M.; Saifullah, A. Low-Power Wide-Area Networks: Opportunities, Challenges, and Directions. In Proceedings of the Workshop Program of the 19th International Conference on Distributed Computing and Networking, Varanasi, India, 4-7 January 2018; p. 8.

15. Vejlgaard, B.; Lauridsen, M.; Nguyen, H.; Kovacs, I.Z.; Mogensen, P.; Sorensen, M. Coverage and Capacity Analysis of Sigfox, LoRa, GPRS, and NB-IoT. In Proceedings of the 2017 IEEE 85th Vehicular Technology Conference (VTC Spring), Sydney, NSW, Australia, 4-7 June 2017.

16. Morin, E.; Maman, M.; Guizzetti, R.; Duda, A. Comparison of the Device Lifetime in Wireless Networks for the Internet of Things. IEEE Access 2017, 5, 7097-7114. [CrossRef]

17. Proskochylo, A.; Vorobyov, A.; Zriakhov, M.; Kravchuk, A.; Akulynichev, A.; Lukin, V. Overview of wireless technologies for organizing sensor networks. In Proceedings of the 2015 Second International Scientific-Practical Conference Problems of Infocommunications Science and Technology (PIC S\&T), Kharkiv, Ukraine, 13-15 October 2015; pp. 39-41.

18. Yin, Y.; Zeng, Y.; Chen, X.; Fan, Y. The internet of things in healthcare: An overview. J. Ind. Inf. Integr. 2016, 1, 3-13. [CrossRef]

19. Rghioui, a.; L'Aarje, A.; Elouaai, F.; Bouhorma, M. The Internet of Things for Healthcare Monitoring: Security Review and Proposed Solution. In Proceedings of the 2014 Third IEEE International Colloquium in Information Science and Technology (CIST), Tetouan, Morocco, 20-22 October 2014; pp. 384-389.

20. Hindia, M.N.; Rahman, T.A.; Ojukwu, H.; Hanafi, E.B.; Fattouh, A. Enabling Remote Health-Caring Utilizing IoT Concept over LTE-Femtocell Networks. PLoS ONE 2016, 11, e0155077. [CrossRef] [PubMed]

21. Fortino, G.; Giannantonio, R.; Gravina, R.; Kuryloski, P.; Jafari, R. Enabling effective programming and flexible management of efficient body sensor network applications. IEEE Trans. Hum. Mach. Syst. 2013, 43, 115-133. [CrossRef]

22. Fortino, G.; Galzarano, S.; Gravina, R.; Li, W. A framework for collaborative computing and multi-sensor data fusion in body sensor networks. Inf. Fus. 2015, 22, 50-70. [CrossRef]

23. Rahmani, A.M.; Gia, T.N.; Negash, B.; Anzanpour, A.; Azimi, I.; Jiang, M.; Liljeberg, P. Exploiting smart e-Health gateways at the edge of healthcare Internet-of-Things: A fog computing approach. Futur. Gener. Comput. Syst. 2018, 78, 641-658. [CrossRef]

24. Rahmani, A.-M.; Thanigaivelan, N.K.; Gia, T.N.; Granados, J.; Negash, B.; Liljeberg, P.; Tenhunen, H. Smart e-Health Gateway: Bringing intelligence to Internet-of-Things based ubiquitous healthcare systems. In Proceedings of the 2015 12th Annual IEEE Consumer Communications and Networking Conference (CCNC), Las Vegas, NV, USA, 9-12 January 2015; pp. 826-834.

25. Nguyen Gia, T.; Dhaou, I.B.; Ali, M.; Rahmani, A.M.; Westerlund, T.; Liljeberg, P.; Tenhunen, H. Energy efficient fog-assisted IoT system for monitoring diabetic patients with cardiovascular disease. Futur. Gener. Comput. Syst. 2019, 93, 198-211. [CrossRef]

26. Shebi Ahammed, S.; Pillai, B.C. Design of Wi-Fi Based Mobile Electrocardiogram Monitoring System on Concerto Platform. Procedia Eng. 2013, 64, 65-73. [CrossRef]

27. Spanï $i \frac{1}{2}$, E.; Di Pascoli, S.; Iannaccone, G. Low-Power Wearable ECG Monitoring System for Multiple-Patient Remote Monitoring. IEEE Sens. J. 2016, 16, 5452-5462. [CrossRef]

28. Singh, M.; Jain, N. Performance and evaluation of smartphone based wireless blood pressure monitoring system using Bluetooth. IEEE Sens. J. 2016, 16, 8322-8328. [CrossRef]

29. Lee, H.C.; Ke, K.H. Monitoring of Large-Area IoT Sensors Using a LoRa Wireless Mesh Network System: Design and Evaluation. IEEE Trans. Instrum. Meas. 2018, 67, 2177-2187. [CrossRef]

30. Mdhaffar, A.; Chaari, T.; Larbi, K.; Jmaiel, M.; Freisleben, B. IoT-based health monitoring via LoRaWAN. In Proceedings of the IEEE EUROCON 2017 17th International Conference on Smart Technologies, Ohrid, Macedonia, 6-8 July 2017; pp. 519-524.

31. Petäjäjärvi, J.; Mikhaylov, K.; Yasmin, R.; Hämäläinen, M.; Iinatti, J. Evaluation of LoRa LPWAN Technology for Indoor Remote Health and Wellbeing Monitoring. Int. J. Wirel. Inf. Netw. 2017, 24, 153-165. [CrossRef]

32. Sodhro, A.H.; Chen, L.; Sekhari, A.; Ouzrout, Y.; Wu, W. Energy efficiency comparison between data rate control and transmission power control algorithms for wireless body sensor networks. Int. J. Distrib. Sens. Netw. 2018, 14. [CrossRef]

33. Frustaci, M.; Pace, P.; Aloi, G.; Fortino, G. Evaluating critical security issues of the IoT world: Present and future challenges. IEEE Internet Things J. 2018, 5, 2483-2495. [CrossRef] 
34. Aras, E.; Ramachandran, G.S.; Lawrence, P.; Hughes, D. Exploring the security vulnerabilities of LoRa. In Proceedings of the 2017 3rd IEEE International Conference on Cybernetics (CYBCONF), Exeter, UK, 21-23 June 2017.

35. Satija, U.; Ramkumar, B.; Manikandan, S.M. Real-Time Signal Quality-Aware ECG Telemetry System for IoT-Based Health Care Monitoring. IEEE Internet Things J. 2017, 4, 815-823. [CrossRef]

36. Zhang, Y.; Xiao, H. Bluetooth-based sensor networks for remotely monitoring the physiological signals of a patient. IEEE Trans. Inf. Technol. Biomed. 2009, 13, 1040-1048. [CrossRef] [PubMed]

37. Kim, Y.; Lee, S.S.; Lee, S.K. Coexistence of ZigBee-based WBAN and WiFi for health telemonitoring systems. IEEE J. Biomed. Heal. Inform. 2016, 20, 222-230. [CrossRef] [PubMed]

38. Tan, B.; Chen, Q.; Chetty, K.; Woodbridge, K.; Li, W.; Piechocki, R. Exploiting WiFi Channel State Information for Residential Healthcare Informatics. IEEE Commun. Mag. 2018, 56, 130-137. [CrossRef]

39. Xu, J.Y.; Wang, Y.; Barrett, M.; Dobkin, B.; Pottie, G.J.; Kaiser, W.J. Personalized multilayer maily life profiling through context enabled activity classification and motion reconstruction: An integrated system approach. IEEE J. Biomed. Heal. Inform. 2016, 20, 177-188. [CrossRef] [PubMed]

40. Wu, F.; Wu, T.; Yuce, M.R. An internet-of-things (IoT) network system for connected safety and health monitoring applications. Sensors (Switzerland) 2019, 19, 21. [CrossRef]

41. Chen, M.; Ma, Y.; Song, J.; Lai, C.F.; Hu, B. Smart Clothing: Connecting Human with Clouds and Big Data for Sustainable Health Monitoring. Mob. Netw. Appl. 2016, 21, 825-845. [CrossRef]

(C) 2019 by the authors. Licensee MDPI, Basel, Switzerland. This article is an open access article distributed under the terms and conditions of the Creative Commons Attribution (CC BY) license (http://creativecommons.org/licenses/by/4.0/). 